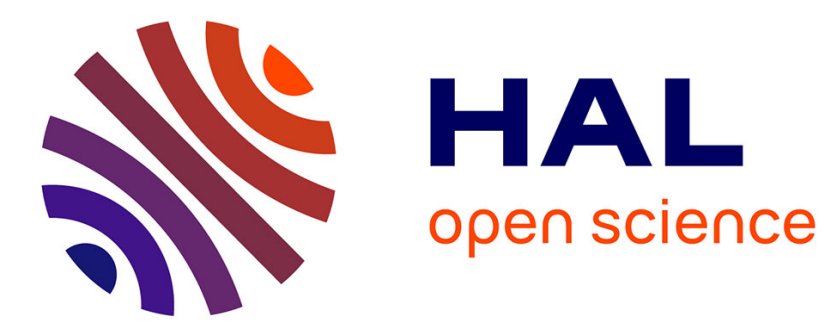

\title{
Stochastic linearization method with random parameters for SDOF nonlinear dynamical systems: Prediction and identification procedures
}

Christian Soize

\section{- To cite this version:}

Christian Soize. Stochastic linearization method with random parameters for SDOF nonlinear dynamical systems: Prediction and identification procedures. Probabilistic Engineering Mechanics, 1995, 10 (3), pp.143-152. 10.1016/0266-8920(95)00011-M . hal-00770288

\section{HAL Id: hal-00770288 \\ https://hal.science/hal-00770288}

Submitted on 4 Jan 2013

HAL is a multi-disciplinary open access archive for the deposit and dissemination of scientific research documents, whether they are published or not. The documents may come from teaching and research institutions in France or abroad, or from public or private research centers.
L'archive ouverte pluridisciplinaire HAL, est destinée au dépôt et à la diffusion de documents scientifiques de niveau recherche, publiés ou non, émanant des établissements d'enseignement et de recherche français ou étrangers, des laboratoires publics ou privés. 


\title{
Stochastic linearization method with random parameters for SDOF nonlinear dynamical systems: prediction and identification procedures
}

\author{
C. Soize \\ Structures Department, Office National d'Études et de Recherches Aérospatiales (ONERA), \\ BP 72, 92322 Chatillon Cedex, France
}

This paper describes firstly, the calculation of the Power Spectral Density Function (PSDF) for the stationary response of SDOF nonlinear second-order dynamical systems excited by a white or a broad-band gaussian noise, and secondly, the identification of a single-degree-of-freedom (SDOF) nonlinear dynamical second-order dynamical system driven by a broad-band or a colored gaussian noise. The two aspects are based on the use of a stochastic linearization method with random parameters which is an efficient way of approximating the PSDF. The gain obtained by this method is shown on a SDOF nonlinear dynamical system. In addition, it is shown that the stochastic linearization method with random parameters is an efficient approach for identifying a SDOF nonlinear dynamical system.

\section{INTRODUCTION}

Our purpose here is 1)- to calculate the Power Spectral Density Function (PSDF) for the stationary response of single-degree-of-freedom (SDOF) nonlinear random oscillators subjected to a white or a broad-band Gaussian noise and 2)- to present an identification procedure of a SDOF nonlinear second-order dynamical system driven by a broad-band or a Gaussian colored noise.

The statistical linearization method ${ }^{1-7}$ is quite general in the sense that the PSDF can be calculated for multidimensional nonlinear dynamical systems. Unfortunately, when strong non-linear effects exist, this method, while still yielding correct approximations of the root mean-square of responses, overestimates the peak values of the PSDF and underestimates the spectral bandwidths ${ }^{8-12}$

There are many methods to determine approximate expressions of the PSDF, but there only a few general comments about these approximate methods will be given.

The stochastic averaging method, initially developed by Stratonovich ${ }^{13}$, is an effective approximate method for predicting stochastic response of SDOF nonlinear dynamical systems subject to broadband random excitations. This method has been applied extensively to the field of mechanics ${ }^{14-19}$. Recently, Bouc ${ }^{12}$ proposed another method for constructing an approximation of the PSDF of weakly damped, strongly nonlinear random oscillators. This method is based on an equivalent linear system with coefficients depending on the amplitude process of the nonlinear oscillations, and the amplitude process is derived by the averaging stochastic method.

The Fokker-Planck equation method theoretically allows the construction of the matrix-valued spectral density function to be obtained for the stationary response of multidimensional secondorder nonlinear random oscillators driven by vector-valued Gaussian white noise ${ }^{20}$. For such systems exact expressions for spectra are nonexistent. However, it should be noted that an exact expression has been obtained ${ }^{9,20}$ for the matrix-valued spectral density function of multidimensional linear oscillators subjected to an external vector-valued Gaussian broad-band noise and an additive 
parametric stochastic excitation represented by a matrix-valued Gaussian broad-band noise. Starting from this kind of approach, Valéry Roy $\& \operatorname{Spanos}^{11}$ developed an interesting approximate expression of PSDF for a SDOF nonlinear dynamical system based on a recursion method.

Within the context of nonlinear dynamical system identification, a general methodology and practical techniques for analyzing a wide class of nonlinear systems consisting of parallel linear, bilinear and trilinear systems, have been studied by Bendat ${ }^{21}$.

For many years, numerous publications have appeared on methods for solving this type of problem, but it is clear that the greatest difficulty is trying to extend them to multidimensional cases for prediction and identification problems. Except for the Monte Carlo numerical simulation method ${ }^{22}$, which can solve practically any nonlinear stochastic problem, though with a high cost for large multidimensional nonlinear systems, only the statistical linearization method is really efficient at the present time for nonlinear stochastic systems with many degrees of freedom. Thus the incentive is great to extend the classical statistical linearization method to improve the prediction of the matrix-valued spectral density function for those cases where this method fails. In addition and within this context, the problems related to nonlinear system identification are important.

To improve the linearization method, Crandall ${ }^{23}$ and Miles $^{8}$ developed a heuristic approach for estimating PSDF, based on the concept of an equivalent linear system with random parameters.

This paper deals with a stochastic linearization method with random parameters whose mathematical aspects were discussed in $1991^{9}$ and main ideas shortly presented in $1994^{10}$. The adopted approach is an alternative method for calculating the PSDF of a SDOF nonlinear dynamical system driven by a white or a broad-band Gaussian noise. The paper also presents new results within the context of nonlinear dynamical system identification. It is shown that the stochastic linearization method with random parameters is an efficient approach for identifying a SDOF nonlinear second-order dynamical system driven by a broad-band or a colored gaussian noise.

The present developments are limited to the case of SDOF nonlinear dynamical systems. The extension to multidimensional cases is in $\operatorname{progress}^{27}$.

\section{NONLINEAR STOCHASTIC DIFFERENTIAL EQUATION OF THE PROBLEM}

Consider the following SDOF nonlinear stochastic second-order dynamical system:

$$
M \ddot{Q}(t)+2 \xi M \omega_{0} \dot{Q}(t)+M \omega_{0}^{2} Q(t)+\varepsilon f(Q(t), \dot{Q}(t))=g_{0} X(t),
$$

where $M, \xi, \omega_{0}$ and $g_{0}$ are greater than zero, $\varepsilon \geq 0,(q, \dot{q}) \mapsto f(q, \dot{q})$ is a real-valued function on $\mathbb{R} \times \mathbb{R}$. The stochastic process $X$ is either a real-valued second-order stationary Gaussian process indexed by $\mathbb{R}$, centered, mean-square continuous and having a power spectral density function $\omega \mapsto S_{X}(\omega)$ (which is then integrable on $\mathbb{R}$ ), or a normalized Gaussian white noise with power spectral density function $S_{X}(\omega)=1 / 2 \pi$ for all real $\omega$. In the last case, $X$ is denoted by $\dot{W}$. It is assumed that the function $f$ verifies the necessary conditions for Eq. (1) to have a unique stationary solution denoted as $Q$, which is a centered second-order stochastic process on $\mathbb{R}$. Let $\omega \mapsto S_{Q}(\omega)$ be its PSDF. Finally, one denotes as $M_{\mu, \nu}$ the moments defined by

$$
\mathbb{M}_{\mu, \nu}=E\left(Q(t)^{\mu} \dot{Q}(t)^{\nu}\right)=\int_{\mathbb{R}} \int_{\mathbb{R}} q^{\mu} \dot{q}^{\nu} p_{Q(t), \dot{Q}(t)}(q, \dot{q}) d q d \dot{q}
$$

where $p_{Q(t), \dot{Q}(t)}(q, \dot{q}) d q d \dot{q}$ is the probability law of the random variable $(Q(t), \dot{Q}(t))$ on $\mathbb{R}^{2}$ for all fixed $t$. Therefore, one has $M_{1,0}=M_{0,1}=M_{1,1}=0$ and $M_{2,0}<+\infty, M_{0,2}<+\infty$. 


\section{LINEAR STOCHASTIC DIFFERENTIAL EQUATION WITH RANDOM PARAMETERS FOR CONSTRUCTING AN EQUIVALENT SYSTEM}

The linear stochastic differential equation with random parameters that will be used to approximate Eq. (1) is written

$$
M \ddot{Y}(t)+2 \xi M \omega_{0}(1+\varepsilon \Theta) \dot{Y}(t)+M \omega_{0}^{2}(1+\varepsilon \Lambda) Y(t)=g_{0} X(t)
$$

where $M, \xi, \omega_{0}, g_{0}, \varepsilon$ and $X$ are the previously defined quantities. The $\mathbb{R}^{2}$-vector $(\Theta, \Lambda)$ is a random variable, independent of the process $X$, and its probability law $P_{\Theta, \Lambda}(d \theta, d \lambda)$ on $\mathbb{R}^{2}$ verifies the following property concerning its support :

$$
\operatorname{Supp} P_{\Theta, \Lambda} \subset\left[\theta_{0},+\infty\left[\times\left[\lambda_{0},+\infty[,\right.\right.\right.
$$

where $\theta_{0}$ and $\lambda_{0}$ are any real constants verifying $1+\varepsilon \theta_{0}>0$ and $1+\varepsilon \lambda_{0}>0$. In addition, one assumes that probability law $P_{\Theta, \Lambda}(d \theta, d \lambda)$ verifies the inequalities

$$
\int_{\mathbb{R}} \int_{\mathbb{R}} \frac{P_{\Theta, \Lambda}(d \theta, d \lambda)}{(1+\varepsilon \theta)(1+\varepsilon \lambda)}<+\infty \quad, \quad \int_{\mathbb{R}} \int_{\mathbb{R}} \frac{P_{\Theta, \Lambda}(d \theta, d \lambda)}{(1+\varepsilon \theta)}<+\infty .
$$

Let us note that this model allows us to write $P_{\Theta, \Lambda}(d \theta, d \lambda)=\delta_{0} \otimes P_{\Lambda}(d \lambda)$ or $P_{\Theta, \Lambda}(d \theta, d \lambda)=$ $P_{\Theta}(d \theta) \otimes \delta_{0}$, where $\delta_{0}$ is the Dirac measure at the origin of $\mathbb{R}$. Consequently, one can use a model with only one random parameter (stiffness or damping), or with two random parameters (stiffness and damping). With the hypotheses defined by Eqs. (4) and (5), it can be proved ${ }^{9}$ that Eq. (3) has a second-order stationary solution $Y$. For all $t$ fixed in $\mathbb{R}$, the $\mathbb{R}^{2}$-valued random variable $(Y(t), \dot{Y}(t))$ has a non gaussian probability density function $\left.p_{Y(t), \dot{Y}(t)}(y, \dot{y})\right)$ which is explicitly known ${ }^{9}$, and the PSDF of process $Y$ can be written as

$$
S_{Y}(\omega)=\frac{4 \xi C_{0}}{\omega_{0}} S_{X}(\omega) \int_{\mathbb{R}} \int_{\mathbb{R}} \frac{P_{\Theta, \Lambda}(d \theta, d \lambda)}{\left\{\left(1+\varepsilon \lambda-\left(\frac{\omega}{\omega_{0}}\right)^{2}\right)^{2}+4 \xi^{2}\left(\frac{\omega}{\omega_{0}}\right)^{2}(1+\varepsilon \theta)^{2}\right\}},
$$

in which

$$
C_{0}=g_{0}^{2} /\left(4 \xi M^{2} \omega_{0}^{3}\right)
$$

\subsection{Expressions for the moments of a Gaussian colored excitation}

Let $\mathcal{M}_{\mu, \nu}$ be the moments defined by

$$
\mathcal{M}_{\mu, \nu}=E\left(Y(t)^{\mu} \dot{Y}(t)^{\nu}\right)=\int_{\mathbb{R}} \int_{\mathbb{R}} y^{\mu} \dot{y}^{\nu} p_{Y(t), \dot{Y}(t)}(y, \dot{y}) d y d \dot{y}
$$

Since $Y$ is a centered stationary process, one has

$$
\mathcal{M}_{1,0}=E\{Y(t)\}=0 \quad, \quad \mathcal{M}_{0,1}=E\{\dot{Y}(t)\}=0 \quad, \quad \mathcal{M}_{1,1}=E\{Y(t) \dot{Y}(t)\}=0 \quad .
$$

Moments $\mathcal{M}_{2,0}=E\left\{Y(t)^{2}\right\}$ and $\mathcal{M}_{0,2}=E\left\{\dot{Y}(t)^{2}\right\}$ are given by the formulas

$$
\mathcal{M}_{2,0}=\int_{\mathbb{R}} S_{Y}(\omega) d \omega \quad, \quad \mathcal{M}_{0,2}=\int_{\mathbb{R}} \omega^{2} S_{Y}(\omega) d \omega
$$

in which $S_{Y}(\omega)$ is defined by Eq. (6). 


\subsection{Expression for the moments of a Gaussian white excitation}

In this case, for all real $\omega$ one has $S_{X}(\omega)=1 / 2 \pi$ and Eqs. (5), (6) and (10) yield ${ }^{9}$

$$
\begin{gathered}
\mathcal{M}_{2,0}=E\left\{Y(t)^{2}\right\}=C_{0} \int_{\mathbb{R}} \int_{\mathbb{R}} \frac{P_{\Theta, \Lambda}(d \theta, d \lambda)}{(1+\varepsilon \theta)(1+\varepsilon \lambda)}<+\infty, \\
\mathcal{M}_{0,2}=E\left(\dot{Y}(t)^{2}\right)=\omega_{0}^{2} C_{0} \int_{\mathbb{R}} \frac{P_{\Theta}(d \theta)}{1+\varepsilon \theta}<+\infty .
\end{gathered}
$$

\section{PREDICTION OF THE PSDF USING A STOCHASTIC LINEARIZATION METHOD WITH RANDOM PARAMETERS}

In this section, the nonlinear system being driven by a Gaussian white noise, one has $X=\dot{W}$ and then $S_{X}(\omega)=1 / 2 \pi$ for all $\omega$ in $\mathbb{R}$. The stochastic linearization method with random parameters consists in constructing the probability measure $P_{\Theta, \Lambda}(d \theta, d \lambda)$ in such a way as to minimize the distance between the stationary stochastic processes $Q$ and $Y$ verifying Eq. (1) and Eq. (3) respectively. There are several possible choices for this distance.

- Firstly, it can easily be verified ${ }^{9}$ that processes $Q$ and $Y$ cannot in general be stochastically equivalent in the wide sense, i.e. no probability measure $P_{\Theta, \Lambda}$ exists such that processes $Q$ and $Y$ have identical systems of marginal probability laws.

- Secondly, it can be proved ${ }^{9}$ that, if equality $P_{Y(t), \dot{Y}(t)}=P_{Q(t), \dot{Q}(t)}$ of marginal probability laws is used, then the probability measure $P_{\Theta, \Lambda}$ must be a solution of the following integral equation on $\mathbb{R}^{2}$ :

$$
\int_{\mathbb{R}} \int_{\mathbb{R}} p_{Y_{\theta, \lambda}(t), \dot{Y}_{\theta, \lambda}(t)}(q, \dot{q} \mid \theta, \lambda) P_{\Theta, \Lambda}(d \theta, d \lambda)=p_{Q(t), \dot{Q}(t)}(q, \dot{q})
$$

where conditional probability density function $p_{Y_{\theta, \lambda}(t), \dot{Y}_{\theta, \lambda}(t)}(q, \dot{q} \mid \theta, \lambda)$ is an explicitly known Gaussian probability density function ${ }^{9}$. Existence of a solution verifying Eqs. (4), (5) and (13) is a problem which is still open.

- Thirdly, the equality of a finite number of moments can be written ${ }^{9}$ and consequently, a finite number of integral equations defined by these equalities must be solved. This problem is close to the second one and is related to the representation of $P_{\Theta, \Lambda}$ as it is developed by Soize ${ }^{9}$.

- Finally, as the aim of the prediction is to construct the PSDF of the stationary response, it is quite natural to determine $P_{\Theta, \Lambda}$ by writing the equality of the second-order moments

$$
M_{2,0}=\mathcal{M}_{2,0} \quad, \quad M_{0,2}=\mathcal{M}_{0,2} \quad,
$$

and obviously, the normalization condition. So, one obtains the following formulation ${ }^{9,10}$.

\subsection{Problem to be solved}

From Eq. (14), we deduce that probability measure $P_{\Theta, \Lambda}(d \theta, d \lambda)$ on $\mathbb{R}^{2}$, having to verify condition (4), is a solution of the following integral equations

$$
\int_{\mathbb{R}} \int_{\mathbb{R}} \frac{P_{\Theta, \Lambda}(d \theta, d \lambda)}{(1+\varepsilon \theta)(1+\varepsilon \lambda)}=\mathcal{S}_{2,0} \quad, \quad \int_{\mathbb{R}} \int_{\mathbb{R}} \frac{P_{\Theta, \Lambda}(d \theta, d \lambda)}{(1+\varepsilon \theta)}=\mathcal{S}_{0,2},
$$

where $\mathcal{S}_{2,0}$ and $\mathcal{S}_{0,2}$ are the positive constants defined by

$$
\mathcal{S}_{2,0}=\frac{M_{2,0}}{C_{0}} \quad, \quad \mathcal{S}_{0,2}=\frac{M_{0,2}}{\omega_{0}^{2} C_{0}} .
$$

A priori, the solution of this problem is not unique. Consequently, one can define a subset of probability laws such that there exists a unique solution $P_{\Theta, \Lambda}(d \theta, d \lambda)$ of Eq. (15) in this subset. 


\subsection{Definition of a subset $\mathcal{P}$ of probability laws on $\mathbb{R}$}

One defines the subset $\mathcal{P}$ of probability laws $P_{X}(d x)=p_{X}(x) d x$ on $\mathbb{R}$, having parameters $x_{0} \in \mathbb{R}$, $\alpha>0$ and $\beta>0$ and such that for all $x \in \mathbb{R}$

$$
\begin{gathered}
p_{X}(x)=\alpha(1+\varepsilon x) W_{X}(x), \\
W_{X}(x)=\mathbf{1}_{\left[x_{0},+\infty[\right.}(x)\left(x-x_{0}\right) e^{-\beta\left(x-x_{0}\right)^{2}} .
\end{gathered}
$$

This first-order finite expansion (17) in orthogonal polynomials with respect to the weight $W_{X}$, implies that $x \mapsto p_{X}(x)$ is a continuous function defined on $\mathbb{R}$ with support $\left[x_{0},+\infty\right.$ [ and with all the mth-order moments finite. The following results can be proved ${ }^{9}$ for the three types of modeling corresponding to the cases where the nonlinear dynamical system has a nonlinear stiffness, a nonlinear damping or a nonlinear stiffness and damping.

\subsection{Approximation of the PSDF for a SDOF nonlinear dynamical system driven by a Gaussian white noise}

Case 1: Nonlinear stiffness. If $\mathcal{S}_{0,2}=1$, i.e. if $M_{0,2}=\omega_{0}^{2} C_{0}$, then one can write $P_{\Theta, \Lambda}(d \theta, d \lambda)=$ $\delta_{0} \otimes P_{\Lambda}(d \lambda)$ and choose $P_{\Lambda}(d \lambda)$ in $\mathcal{P}$ with parameters $\lambda_{0}>-1 / \varepsilon, \alpha_{\Lambda}>0, \beta_{\Lambda}>0$ :

$$
P_{\Lambda}(d \lambda)=\alpha_{\Lambda}(1+\varepsilon \lambda) \mathbf{1}_{\left[\lambda_{0},+\infty[\right.}(\lambda)\left(\lambda-\lambda_{0}\right) e^{-\beta_{\Lambda}\left(\lambda-\lambda_{0}\right)^{2}} d \lambda .
$$

For all $\mathcal{S}_{2,0}>0$, if $\lambda_{0}$ is chosen such that $0<1+\varepsilon \lambda_{0}<\mathcal{S}_{2,0}^{-1}$, then the problem defined in Section 4.1 has a unique solution ${ }^{9}$ which is given by Eq. (19) with

$$
\beta_{\Lambda}=\frac{\pi \varepsilon^{2}}{4}\left(\frac{\mathcal{S}_{2,0}}{\mathcal{S}_{0,2}-\mathcal{S}_{2,0}\left(1+\varepsilon \lambda_{0}\right)}\right)^{2} \quad, \quad \alpha_{\Lambda}=2 \beta_{\Lambda} \frac{\mathcal{S}_{2,0}}{\mathcal{S}_{0,2}},
$$

in which $\mathcal{S}_{0,2}=1$. Since $S_{X}(\omega)=1 / 2 \pi$ for all real $\omega$, approximation $S_{Y}(\omega)$ given by Eq. (6) of PSDF $S_{Q}(\omega)$ can be written as

$$
S_{Y}(\omega)=\frac{4 \xi C_{0}}{\pi \omega_{0}} \mathcal{S}_{2,0} \int_{0}^{+\infty} \frac{x\left(1+\varepsilon \lambda_{0}+A x\right) e^{-x^{2}} d x}{\left\{\left(1+\varepsilon \lambda_{0}+A x-\left(\frac{\omega}{\omega_{0}}\right)^{2}\right)^{2}+4 \xi^{2}\left(\frac{\omega}{\omega_{0}}\right)^{2}\right\}},
$$

in which $A=2 \pi^{-1 / 2}\left(\mathcal{S}_{2,0}^{-1}-\left(1+\varepsilon \lambda_{1}\right)\right)>0$. It should be noted that:

(i)- If $\mathcal{S}_{2,0}<1$, then one can choose $\lambda_{0}=0$.

(ii)- If $\mathcal{S}_{2,0}=1$, i.e. if $\varepsilon=0$, then $\lambda_{0}=A=0$.

(iii)- If $\mathcal{S}_{2,0}>1$, then it is necessary to choose $\lambda_{0}<0$ such that $0<1+\varepsilon \lambda_{0}<\mathcal{S}_{2,0}^{-1}$. In fact, the lower bound $\lambda_{0}=-1 / \varepsilon$ can also be used.

Case 2: Nonlinear damping. If $\mathcal{S}_{0,2}=\mathcal{S}_{2,0}$, i.e. if $M_{0,2}=\omega_{0}^{2} M_{2,0}$, then one can write $P_{\Theta, \Lambda}(d \theta, d \lambda)=P_{\Theta}(d \theta) \otimes \delta_{0}$ and choose $P_{\Theta}(d \theta)$ in $\mathcal{P}$ with parameters $\theta_{0}>-1 / \varepsilon, \alpha_{\Theta}>0$, $\beta_{\Theta}>0$ :

$$
P_{\Theta}(d \theta)=\alpha_{\Theta}(1+\varepsilon \theta) \mathbf{1}_{\left[\theta_{0},+\infty[\right.}(\theta)\left(\theta-\theta_{0}\right) e^{-\beta_{\Theta}\left(\theta-\theta_{0}\right)^{2}} d \theta
$$

For all $\mathcal{S}_{0,2}>0$, if $\theta_{0}$ is chosen such that $0<1+\varepsilon \theta_{0}<\mathcal{S}_{0,2}^{-1}$, then the problem defined in Section 4.1 has a unique solution ${ }^{9}$ which is given by Eq. (22), with

$$
\beta_{\Theta}=\frac{\pi \varepsilon^{2}}{4}\left(\frac{\mathcal{S}_{0,2}}{1-\mathcal{S}_{0,2}\left(1+\varepsilon \theta_{0}\right)}\right)^{2} \quad, \quad \alpha_{\Theta}=2 \beta_{\Theta} \mathcal{S}_{0,2} .
$$

Proba. Eng. Mech. 
Since $S_{X}(\omega)=1 / 2 \pi$ for all real $\omega$, the approximation $S_{Y}(\omega)$ given by Eq. (6) of the PSDF $S_{Q}(\omega)$ can be written as

$$
S_{Y}(\omega)=\frac{4 \xi C_{0}}{\pi \omega_{0}} \mathcal{S}_{0,2} \int_{0}^{+\infty} \frac{y\left(1+\varepsilon \theta_{0}+B y\right) e^{-y^{2}} d y}{\left\{\left(1-\left(\frac{\omega}{\omega_{0}}\right)^{2}\right)^{2}+4 \xi^{2}\left(\frac{\omega}{\omega_{0}}\right)^{2}\left(1+\varepsilon \theta_{0}+B y\right)^{2}\right\}},
$$

in which $B=2 \pi^{-1 / 2}\left(\mathcal{S}_{0,2}^{-1}-\left(1+\varepsilon \theta_{0}\right)\right)>0$. It should be noted that:

(i)- If $\mathcal{S}_{2,0}=\mathcal{S}_{0,2}<1$, then one can choose $\theta_{0}=0$.

(ii)- If $\mathcal{S}_{2,0}=\mathcal{S}_{0,2}=1$, i.e. if $\varepsilon=0$, then $\theta_{0}=B=0$.

(iii)- If $\mathcal{S}_{2,0}=\mathcal{S}_{0,2}>1$, then it is necessary to choose $\theta_{0}<0$ such that $0<1+\varepsilon \theta_{0}<\mathcal{S}_{0,2}^{-1}$. As in case 1 , the lower bound $\theta_{0}=-1 / \varepsilon$ can also be used.

Case 3: Nonlinear stiffness and damping. If the hypotheses of cases 1 and 2 do not hold, one considers $\Theta$ and $\Lambda$ as independent random variables. Therefore, one writes $P_{\Theta, \Lambda}(d \theta, d \lambda)=$ $P_{\Theta}(d \theta) \otimes P_{\Lambda}(d \lambda)$, with $P_{\Lambda}(d \lambda)$ given by Eq. (19) and $P_{\Theta}(d \theta)$ by Eq. (22). For all $\mathcal{S}_{0,2}>0$ and $\mathcal{S}_{2,0}>0$ such that $\mathcal{S}_{0,2} \neq \mathcal{S}_{2,0}$ and $\mathcal{S}_{0,2} \neq 1$, if $\lambda_{0}$ is chosen such that $0<1+\varepsilon \lambda_{0}<\mathcal{S}_{0,2} \mathcal{S}_{2,0}^{-1}$ and $\theta_{0}$ as in case 2, then the problem defined in Section 4.1 has a unique solution given by Eqs. (19) and (22) with $\beta_{\Lambda}$ and $\alpha_{\Lambda}$ given by Eq. (20), and $\alpha_{\Theta}$ and $\beta_{\Theta}$ by Eq. (23). The approximation $S_{Y}(\omega)$ given by Eq. (6) of the PSDF $S_{Q}(\omega)$ can then written as

$$
S_{Y}(\omega)=\frac{8 \xi C_{0} \mathcal{S}_{2,0}}{\pi \omega_{0}} \int_{0}^{+\infty} \int_{0}^{+\infty} \frac{\left(1+\varepsilon \lambda_{0}+A x\right)\left(1+\varepsilon \theta_{0}+B y\right) x y e^{-x^{2}-y^{2}} d x d y}{\left\{\left(1+\varepsilon \lambda_{0}+A x-\left(\frac{\omega}{\omega_{0}}\right)^{2}\right)^{2}+4 \xi^{2}\left(\frac{\omega}{\omega_{0}}\right)^{2}\left(1+\varepsilon \theta_{0}+B y\right)^{2}\right\}}
$$

with $B$ as in case 2 and $A=2 \pi^{-1 / 2}\left(\mathcal{S}_{0,2} \mathcal{S}_{2,0}^{-1}-\left(1+\varepsilon \lambda_{0}\right)\right)$. It should be noted that:

(i)- If $\mathcal{S}_{2,0} / \mathcal{S}_{0,2}<1$ and $\mathcal{S}_{0,2}<1$, then one can choose $\lambda_{0}=\theta_{0}=0$.

(ii)- If $\mathcal{S}_{2,0}=\mathcal{S}_{0,2}=1$, i.e. if $\varepsilon=0$, then $\lambda_{0}=\theta_{0}=A=B=0$.

(iii)- If $\mathcal{S}_{2,0} / \mathcal{S}_{0,2}>1$ and $\mathcal{S}_{0,2}>1$, then it is necessary to choose $\lambda_{0}<0$ such that $0<1+\varepsilon \lambda_{0}<$ $\mathcal{S}_{0,2} \mathcal{S}_{2,0}^{-1}$ and $\theta_{0}$ as in case 2 . As in cases 1 and 2 , the lower bounds $\lambda_{0}=-1 / \varepsilon \theta_{0}=-1 / \varepsilon$ can also be used.

\subsection{Application: prediction of the PSDF of the stationary response of a Duffing oscillator driven by a Gaussian white noise}

We consider the Duffing oscillator defined by Eq. (1) with $f(q, \dot{q})=M \omega_{0}^{2} q^{3}, X=\dot{W}, M=1$, $\omega_{0}=2 \pi \times 100, g_{0}$ having the values 2,6 or 12 . Two cases are studied:

Case 1: $\xi=0.025$ and $\varepsilon=344514.18$

Case 2: $\xi=0.0025$ and $\varepsilon=34451.418$.

For these two cases, the choice of the value of $\varepsilon$ is such that $\varepsilon C_{0}=1 / 18,0.5$ and 2 for $g_{0}=2,6$ and 12 respectively.

We know ${ }^{5,20}$ that

$$
\mathcal{S}_{2,0}=\gamma_{\varepsilon} U\left(1, \gamma_{\varepsilon}\right) / U\left(0, \gamma_{\varepsilon}\right)<1 \quad, \quad \mathcal{S}_{0,2}=1 \quad,
$$

in which $\gamma_{\varepsilon}=\left(2 \varepsilon C_{0}\right)^{-1 / 2}$ and where $U(a, x)$ is the parabolic cylinder function defined by

$$
U(a, x)=\frac{e^{-\frac{1}{4} x^{2}}}{\Gamma\left(a+\frac{1}{2}\right)} \int_{0}^{+\infty} e^{-x R-\frac{1}{2} R^{2}} R^{a-\frac{1}{2}} d R
$$

Proba. Eng. Mech. 
where $\Gamma(z)$ is the Euler function. For cases 1 and 2, Eq. (26) yields $\mathcal{S}_{2,0}=0.87942,0.57920$ and 0.36596 for $g_{0}=2,6$ and 12 respectively. Figures 1 to 4 give the normalized levels in $\mathrm{dB}$ : $10 \log _{10}\left(S(\omega) / S_{0}\right)$ with $S_{0}=g_{0}^{2} /(2 \pi)$, of the PSDF $S(\omega)$ as a function of frequency expressed in hertz in the band $0 \mathrm{~Hz}-350 \mathrm{~Hz}$. For cases 1 and 2, figures 1 and 2 compare the PSDF $S(\omega)=S_{Q}(\omega)$ (calculated by Monte Carlo numerical simulation of Eq. (1)), with the PSDF $S(\omega)=S_{Q \text {,lin }}(\omega)$ calculated by the statistical linearization method using the explicit invariant measure of the nonlinear system $^{5,20}$. One then has

$$
S_{Q, \operatorname{lin}}(\omega)=C_{0} \frac{2 \xi}{\pi \omega_{0}} \frac{1}{\left\{\left(\Omega_{e}^{2}-\left(\frac{\omega}{\omega_{0}}\right)^{2}\right)^{2}+4 \xi^{2}\left(\frac{\omega}{\omega_{0}}\right)^{2}\right\}},
$$

in which $\Omega_{e}^{2}=1+\varepsilon \lambda_{e}$ and $\lambda_{e}=3 C_{0} \gamma_{\varepsilon} \frac{U\left(2, \gamma_{\varepsilon}\right)}{U\left(0, \gamma_{\varepsilon}\right)}$.

It can be seen in Fig.1-a that the the statistical linearization method yields correct predictions for small-amplitude responses (small amplitude of the excitation $g_{0}=2$ and a not-too-small damping $\xi=0.025)$, but poor predictions when the amplitude of the responses increase, as shown in Figs.1-c and 2-c. For the same cases 1 and 2, figures 3 and 4 compare the PSDF $S(\omega)=S_{Q}(\omega)$ calculated by Monte Carlo numerical simulation of Eq. (1), with the PSDF $S(\omega)=S_{Y}(\omega)$ calculated by the stochastic linearization method with random parameters, i.e. by Eq. (21) with $\lambda_{0}=0$. It can be seen that correct predictions are obtained for small and large amplitude responses.

\section{IDENTIFICATION PROCEDURE BASED ON THE STOCHASTIC LINEARIZATION METHOD WITH RANDOM PARAMETERS}

\subsection{Definition of the identification problem}

In this section, the identification problem under consideration is defined. One considers a SDOF nonlinear stochastic second-order dynamical system, described by an unknown equation of type (1). This means that there is a unique centered stationary response. The excitation $g_{0} X$ of this system is a physically realizable stochastic process which is a real-valued second-order stationary Gaussian process, centered and having a power spectral density function $\omega \mapsto g_{0}^{2} S_{X}(\omega)$ which is known. An experiment is realized. The nonlinear system is subjected to the known stochastic input $g_{0} X$ and trajectories of the stationary stochastic output $Q$ are measured. Using the usual signal theory and signal processing, estimates can be constructed of the power spectral density function $\omega \mapsto S_{Q}(\omega)$ and moments $M_{2,0}=E\left\{Q(t)^{2}\right\}, M_{0,2}=E\left\{\dot{Q}(t)^{2}\right\}$ of the stationary response $Q$. These quantities are such that

$$
\mathbb{M}_{2,0}=\int_{\mathbb{R}} S_{Q}(\omega) d \omega \quad, \quad M_{0,2}=\int_{\mathbb{R}} \omega^{2} S_{Q}(\omega) d \omega .
$$

The identification problem is then defined as the following. One wishes to construct a linear stochastic differential equation with random parameters looking like Eq. (3) in order to minimize the "distance" between the measured power spectral density function $S_{Q}$ and the power spectral density function $S_{Y}$ given by the model. Therefore, the identification procedure concerns the calculation of the unknown constant coefficients such as $M, \xi, \omega_{0}$ and the probability measure of the random parameters. It is clear that the identification procedure requires that the "distance" be mathematically defined (see below).

In order to simplify the presentation, one will limit the developments to the case where the unknown nonlinear physical system has a linear damping and a nonlinear stiffness. Therefore, the model which can be identified is the linear stochastic equation with random parameters:

$$
M \ddot{Y}(t)+C \dot{Y}(t)+K(1+\Lambda) Y(t)=g_{0} X(t)
$$

Proba. Eng. Mech. 
where $M, C=2 \xi M \omega_{0}, K=M \omega_{0}^{2}$ are unknown real positive coefficients and where the probability law $P_{\Lambda}(d \lambda)$ of the real-valued random variable $\Lambda$ belongs to $\mathcal{P}$ (defined in Section 4.2). From Eqs. (3), (6) and (30), we deduce that the power spectral density function $S_{Y}$ of the stationary solution $Y$ of Eq. (30) can be written as

$$
S_{Y}(\omega)=\frac{4 \xi C_{0}}{\omega_{0}} S_{X}(\omega) \int_{\mathbb{R}} \frac{P_{\Lambda}(d \lambda)}{\left\{\left(1+\varepsilon \lambda-\left(\frac{\omega}{\omega_{0}}\right)^{2}\right)^{2}+4 \xi^{2}\left(\frac{\omega}{\omega_{0}}\right)^{2}\right\}},
$$

in which $\omega_{0}=(K / M)^{1 / 2}, \xi=C /\left(2 M \omega_{0}\right), C_{0}$ is given by Eq. (7), where $P_{\Lambda}(d \lambda)$ is defined by Eq. (19) with $\varepsilon=1$ and the parameters $\lambda_{0}, \alpha_{\Lambda}, \beta_{\Lambda}$ are such that (see Section 4.3, case 1): $\lambda_{0}>-1$, $\alpha_{\Lambda}>0$ and $\beta_{\Lambda}>0$. Consequently, one has

$$
P_{\Lambda}(d \lambda)=\alpha_{\Lambda}(1+\lambda) \mathbf{1}_{\left[\lambda_{0},+\infty[\right.}(\lambda)\left(\lambda-\lambda_{0}\right) e^{-\beta_{\Lambda}\left(\lambda-\lambda_{0}\right)^{2}} d \lambda
$$

Normalization condition

$$
\int_{\mathbb{R}} P_{\Lambda}(d \lambda)=1
$$

implies a relation between $\alpha_{\Lambda}, \beta_{\Lambda}$ and $\lambda_{0}$ which can be written as

$$
\alpha_{\Lambda}=h\left(\beta_{\Lambda}, \lambda_{0}\right)=\frac{4 \beta_{\Lambda} \sqrt{\beta_{\Lambda}}}{\sqrt{\pi}+2 \sqrt{\beta_{\Lambda}}\left(1+\lambda_{0}\right)} .
$$

Substituting Eq. (34) into the right-hand side of Eq. (32) yields

$$
P_{\Lambda}\left(d \lambda \mid \beta_{\Lambda}, \lambda_{0}\right)=h\left(\beta_{\Lambda}, \lambda_{0}\right)(1+\lambda) \mathbf{1}_{\left[\lambda_{0},+\infty[\right.}(\lambda)\left(\lambda-\lambda_{0}\right) e^{-\beta_{\Lambda}\left(\lambda-\lambda_{0}\right)^{2}} d \lambda
$$

From Eqs. (31) and (35), it can be seen that $S_{Y}$ depends only on parameters $\beta_{\Lambda}$ and $\lambda_{0}$. Consequently, Eq. (31) can be rewritten as

$$
S_{Y}\left(\omega \mid \beta_{\Lambda}, \lambda_{0}\right)=\frac{4 \xi C_{0}}{\omega_{0}} S_{X}(\omega) \int_{\mathbb{R}} \frac{P_{\Lambda}\left(d \lambda \mid \beta_{\Lambda}, \lambda_{0}\right)}{\left\{\left(1+\varepsilon \lambda-\left(\frac{\omega}{\omega_{0}}\right)^{2}\right)^{2}+4 \xi^{2}\left(\frac{\omega}{\omega_{0}}\right)^{2}\right\}},
$$

in which $P_{\Lambda}\left(d \lambda \mid \beta_{\Lambda}, \lambda_{0}\right)$ is given by Eq. (35).

\subsection{Identification procedure}

The identification procedure is based on a "true" stochastic linearization method with random parameters and has two main steps.

(i)- The first step allows constant coefficients $M, C$ and $K$ in Eq. (30) to be identified using the concept of "true" stochastic linearization method (see below).

(ii)- In the second step, parameters $\beta_{\Lambda}$ and $\lambda_{0}$ of the probability law $P_{\Lambda}\left(d \lambda \mid \beta_{\Lambda}, \lambda_{0}\right)$ are identified in order to minimize the "distance" between the PSDF $S_{Q}$, which is measured, and the PSDF $S_{Y}$ given by the model defined by Eq. (30). This method will be called a "true" stochastic linearization method with random parameters.

Step 1: Identification of constant coefficients using a "true" stochastic linearization method Since the stochastic linearization method is an efficient method for predicting the second-order moments of the stationary response, it is quite natural to use it within the context of an identification 
problem in order to identify the constant coefficients $M, C$ and $K$. The "true" stochastic linearization method starts from the recent works of Kozin ${ }^{24}$ and has been developed by Fillatre ${ }^{25,26}$. In this work ${ }^{25,26}$,Kozin's results have been extended to the case of the identification of multidimensional nonlinear second-order dynamical systems whose mass, damping and stiffness matrices are unknown and excitations are colored noises. In this extension, a second minimization criterion, as well as an iterative algorithm for readjusting the second-order moments, has been introduced in order to improve the proximity of the mass matrices of the linearized and nonlinear systems. In this method, a first minimization problem is defined as

$$
d_{2}(M, C, K)=\min _{(\widehat{M}, \widehat{C}, \widehat{K}) \in \mathcal{E}} d_{2}(\widehat{M}, \widehat{C}, \widehat{K}),
$$

in which the minimization is performed in an appropriate subset $\mathcal{E}$ such that the identified linearized system be asymptotically stable and where $d_{2}$ is the square of the distance such that

$$
d_{2}(\widehat{M}, \widehat{C}, \widehat{K})=E\left\{\left\|g_{0} X(t)-(\widehat{M} \ddot{Q}(t)+\widehat{C} \dot{Q}(t)+\widehat{K} Q(t))\right\|^{2}\right\} \quad .
$$

This method is called a "true" stochastic linearization because it refers to the fact that the mathematical expectation that enters into the right-hand side of Eq. (38) is calculated from the true joint probability law of the random variables $Q(t), \dot{Q}(t), \ddot{Q}(t), g_{0} X(t)$ for all fixed $t$ in $\mathbb{R}$, and is contrasted to Gaussian stochastic linearization. Within the context of the identification procedure, it should be noted that this "true" stochastic linearization is achieved using the signal processing based on the measured trajectories of the input and ouput stochatic processes of the nonlinear systems ${ }^{24,25,26}$ (see the beginning of this section). This means that, since $Q, \dot{Q}, \ddot{Q}$ and $g_{0} X$ are the output and input stationary stochastic processes of the nonlinear dynamical system (output and input are measured), the mathematical expectation in Eq. (38) is estimated without introducing any approximation or additional hypotheses on the joint probability law of random variables $Q(t), \dot{Q}(t), \ddot{Q}(t), g_{0} X(t)$. Herein, this method which is adapted to the multidimensional identification problem, is applied to the SDOF nonlinear system in order to indentify coefficients $M, C$ and $K$. It should be noted that, if the identification was stopped at this step 1 , then one would obtain an identified model which would give correct prediction for the second-order moments (see Section 4.4), but which would give bad predictions for the power spectral density function in some cases (as it is explained in Section 4.4). This is the reason why a second step is included to improve the identification procedure.

Step 2: Final identification step using a "true" stochastic linearization method with random parameters. In this step, coefficients $M, C$ and $K$ in the right-hand side of Eq. (30) are known (identified in step 1). Now, the problem is to identify parameters $\beta_{\Lambda}$ and $\lambda_{0}$ appearing in Eq. (36). Let $\mathcal{D}$ be the subset of $\mathbb{R}^{2}$ such that $\mathcal{D}=\left[0,+\infty[\times]-1,+\infty\left[\right.\right.$. Let $D_{2}\left(\widehat{\beta}_{\Lambda}, \widehat{\lambda}_{0}\right)$ be the square of the distance such that

$$
D_{2}\left(\widehat{\beta}_{\Lambda}, \widehat{\lambda}_{0}\right)=\int_{\mathbb{R}}\left|S_{Y}\left(\omega \mid \widehat{\beta}_{\Lambda}, \widehat{\lambda}_{0}\right)-S_{Q}(\omega)\right|^{2} d \omega
$$

where $S_{Q}$ is the estimated PSDF (from the measurements) and $S_{Y}$ is given by Eq. (36). One then looks into a problem of finding $\left(\beta_{\Lambda}, \lambda_{0}\right) \in \mathcal{D}$ fulfilling the minimum of $D_{2}\left(\widehat{\beta}_{\Lambda}, \widehat{\lambda}_{0}\right)$, i.e.

$$
D_{2}\left(\beta_{\Lambda}, \lambda_{0}\right)=\min _{\left(\widehat{\beta}_{\Lambda}, \widehat{\lambda}_{0}\right) \in \mathcal{D}} D_{2}\left(\widehat{\beta}_{\Lambda}, \widehat{\lambda}_{0}\right)
$$

The constrained optimization problem defined by Eq. (40) is solved using a global minimization algorithm with constraints, based on the used of an iterative method in which an optimization problem of a convex function is solved at each iteration ${ }^{27}$. 


\subsection{Application: identification of a SDOF nonlinear dynamical system driven by a Gaussian colored noise}

In this example, the "experimental data basis" is generated by a Monte Carlo numerical simulation of the Duffing oscillator

$$
M \ddot{Q}(t)+2 \xi M \omega_{0} \dot{Q}(t)+M \omega_{0}^{2} Q(t)+\varepsilon M \omega_{0}^{2} Q(t)^{3}=g_{0} X(t)
$$

Four "experiments" are constructed by numerical simulation:

Case 1: $M=1, \omega_{0}=2 \pi \times 100, \xi=0.025, \varepsilon=344514.18, C=2 \xi M \omega_{0}=31.41, g_{0}=2$ or 6 .

Case 2: $M=1, \omega_{0}=2 \pi \times 100, \xi=0.0025, \varepsilon=34451.418, C=2 \xi M \omega_{0}=3.14, g_{0}=2$ or 6 . For these four cases, $\mathrm{X}$ is a real-valued second-order stationary Gaussian process indexed by $\mathbb{R}$, centered, having a power spectral density function such that for all $\omega$ in $\mathbb{R}$,

$$
S_{X}(\omega)=\frac{1}{2 \pi} \mathbf{1}_{[-2 \pi \times 150,-2 \pi \times 50]}(\omega) \cup \mathbf{1}_{[2 \pi \times 50,2 \pi \times 150]}(\omega) \quad,
$$

in which $\mathbf{1}_{B}(\omega)=1$ if $\omega \in B$ and $=0$ if $\omega \notin B$. Stochastic process $X$ is simulated using a bandpass Butterworth linear filter of order 6, whose lower and upper cutoff frequencies are $50 \mathrm{~Hz}$ and $150 \mathrm{~Hz}$ respectively.

Step 1 and step 2 of the identification procedure yields:

Case $1: M=1 ; C=31.41 ; K=449962$ and $645336, \beta_{\Lambda}=81.79$ and $6.02, \lambda_{0}=-0.106$ and -0.344 for $g_{0}=2$ and 6 , respectively.

Case $2: M=1 ; C=3.14 ; K=452467$ and $647548, \beta_{\Lambda}=51.13$ and $5.25, \lambda_{0}=-0.134$ and -0.346 for $g_{0}=2$ and 6 , respectively.

Figures 5 and 6 give the normalized levels in $\mathrm{dB}: 10 \log _{10}\left(S(\omega) / S_{0}\right)$ with $S_{0}=g_{0}^{2} /(2 \pi)$, of the PSDF $S(\omega)$ as a function of frequency expressed in hertz. For cases 1 and 2, these figures compare the "experimental" PSDF $S(\omega)=S_{Q}(\omega)$ (Monte Carlo numerical simulation of Eq. (41)), with the PSDF $S(\omega)=S_{Y}(\omega)$ calculated with the identification method. It can be seen that a correct identification is obtained for small and large amplitude responses.

\section{CONCLUSIONS}

We have presented a stochastic linearization method with random parameters which allows us to calculate an approximation of the power spectral density functions of stationary responses of SDOF nonlinear second-order dynamical systems.

One has seen that this method yields accurate predictions of the power spectral density function for the stationary reponse of the stochastic Duffing oscillator driven by a Gaussian white noise, whereas the power spectral density function is not correctly predicted in any of the cases by the usual statistical linearization method.

This stochastic linearization method with random parameters has also been used to develop an identification procedure of SDOF nonlinear second-order dynamical systems driven by Gaussian colored noises. The results obtained for the identification of a Duffing oscillator are good enough. Presently, the prediction and identification procedures have been developed only for SDOF nonlinear second-order dynamical systems. The extension of the proposed identification method to the multidimensional cases is possible and is is in progress. 


\section{REFERENCES}

1. Caughey, T.K., Equivalent linearization techniques. Journal of the Acoustical Society America, 1963, 35(11), 1706-1711.

2. Lin, Y.K., Probabilistic Theory of Structural Dynamics. McGraw-Hill, New York, 1967.

3. Crandall, S.H., Heuristic and equivalent linearization techniques for random vibration of nonlinear oscillators. 6th Int. Conf. Nonlinear Oscillations (ICNO), Prague, Czechoslovakia, 1978.

4. Spanos, P.D., Stochastic linearization in structural dynamics. Applied Mechanics Reviews, 1981, 34(1), 1-8.

5. Roberts, J.B. \& Spanos, P.D., Random Vibration and Statistical Linearization. John Wiley and Sons, New York, 1990.

6. Elishakoff I. \& Cai G.Q., Approximate solution for nonlinear random vibration problems by partial stochastic linearization. Probabilistic Engineering Mechanics, 1993, (8), 233-237.

7. Schuëller, G.I., Pandey, M.D. \& Pradlwarter H.J., Equivalent linearization (EQL) in engineering practice for aseismic design. Probabilistic Engineering Mechanics, 1994, (9), 95-102.

8. Miles, R.N., An approximate solution for the spectral response of Duffing's oscillator with random input. Journal of Sound and Vibration, 1989, 132(1), 43-49.

9. Soize, C., Sur le calcul des densités spectrales des réponses stationnaires pour des systèmes dynamiques stochastiques non-linéaires. In Contrôle Actif Vibroacoustique et Dynamique Stochastique, Publications du LMA-CNRS, Marseille, France, ISSN 0750-7356, 1991, pp 297-344.

10. Soize, C., Stochastic linearization method with random parameters and power spectral density calculation, pp 217-222 in Structural Safety and Reliability, Edited by G.I. Schuëller, M. Shinozuka and J.T.P. Yao, Balkema, Rotterdam, Brookfield, 1994.

11. Valéry Roy, R., \& Spanos, P.D., Power spectral density of nonlinear system response: the recursion method. Transactions of the ASME, 1993, 60, 358-365.

12. Bouc, R., The power spectral density of response for a strongly non-linear random oscillation. Journal of Sound and Vibration, 1994, 175(3), 317-331.

13. Stratonovich, R.L., Topics in the Theory of Random Noise, Vol 1. Gordon and Beach, New York, 1963.

14. Iwan, W.D. \& Spanos, P.D., Response envelope statistics for nonlinear oscillators with random excitation. Journal of Applied Mechanics, ASME, 1978, 45.

15. Roberts, J.B., The energy envelope of a randomly excited nonlinear oscillator. Journal of Sound and Vibration, 1978, 60, 177-185.

16. Roberts, J.B. and Spanos, P.D., Stochastic averaging: an approximate method of solving random vibration problems. Int. J. Non-linear Mech., 1986, 21(2), 111-134.

17. Lin, Y.K., Some observations on the stochastic averaging method. Probabilistic Engineering Mechanics, 1986, 1(1), 23-27.

18 Zhu W.Q. , Yu Y.S. \& Lin Y.K., On improved stochastic averaging procedure. Probabilistic Engineering Mechanics, 1994, (9), 203-211.

19. Valéry Roy, R., Stochastic averaging of oscillators excited by colored Gaussian processes. Int. J. Non-Linear Mechanics, 1994, 29(4), 463-475.

20. Soize, C,. The Fokker-Planck Equation for Stochastic Dynamical Systems and its Explicit Steady State Solutions. World Scientific, Singapore, 1994. 
21. Bendat, J.S., Nonlinear System Analysis and Identification From Random Data. John Wiley and Sons, New York, 1990.

22. Shinozuka, M., Simulation of multivariate and multidimensional random processes. Journal of the Acoustical Society America, 1971, 49(1), 357-367.

23. Crandall, S.H., Les vibrations forçées dans les systèmes non linéaires. Colloques internationaux du CNRS, Marseille, France, 1963, 148.

24. Kozin, F., Structural Parameter Identification Techniques, in Analysis and Estimation of Stochastic Mechanical Systems, W. Schiehlen and W. Wedig (ed.), 137-200, SpringerVerlag, New York, 1988.

25. Fillatre, O., Identification des systèmes dynamiques faiblement non-linéaires à partir d'excitations aléatoires. Thèse de doctorat de Mécanique de l'Ecole Centrale Paris, Paris, 26 juin 1991.

26. Fillatre, O., Identification of weakly nonlinear dynamic systems by means of random excitations. La Recherche Aérospatiale, 1992, 3, 11-22.

27. Lefur, O., Identification des systèmes dynamiques multidimensionnels faiblement nonlinéaires par une méthode de linéarisation stochastique à paramètres aléatoires. Thèse de doctorat de Mathématiques de l'Université Pierre et Marie Curie, Paris, Septembre 1995. 

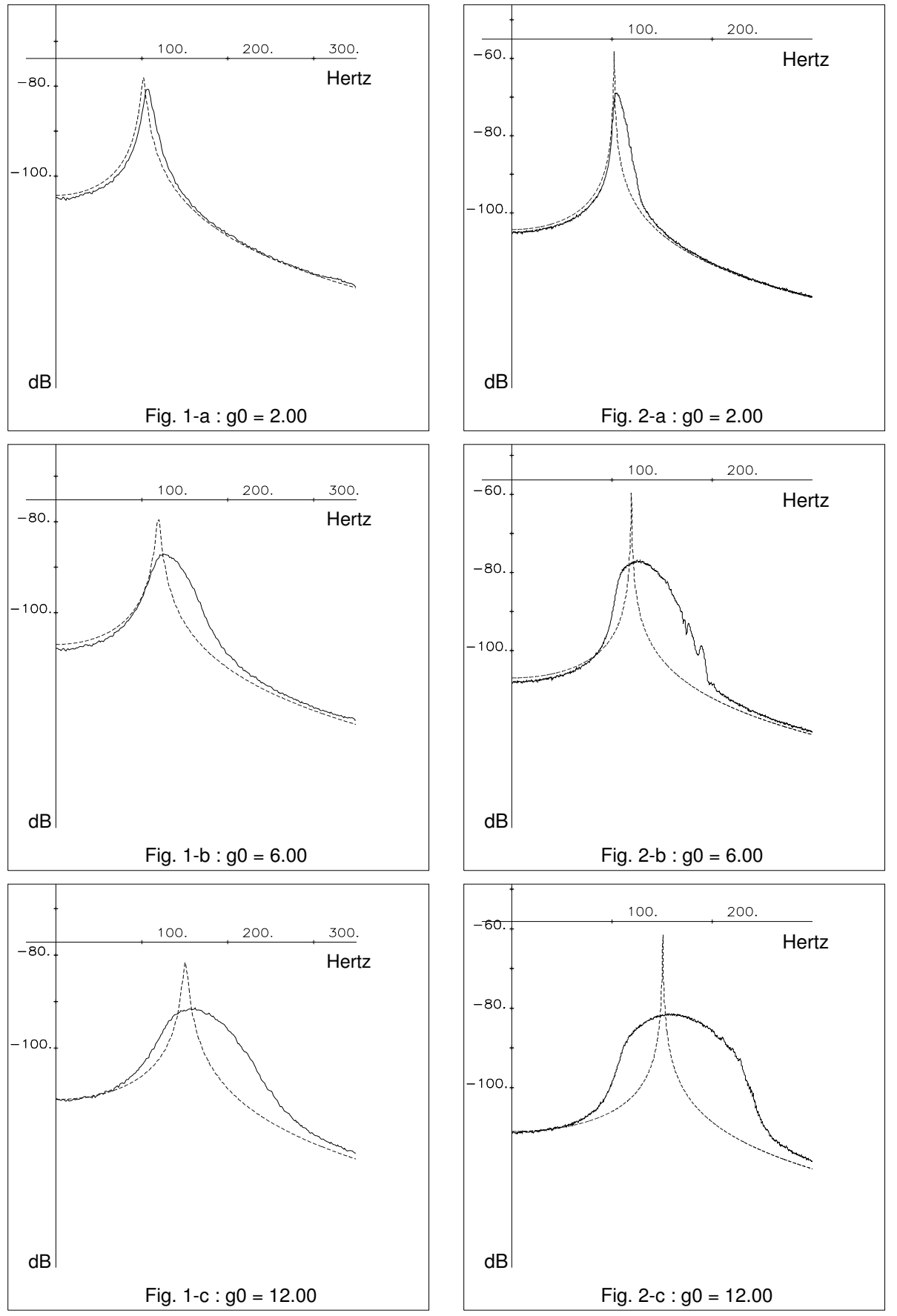

Fig. 1 : CASE 1

Fig. 2 : CASE 2 numerical simulation numerical simulation Classical SLM Classical SLM 

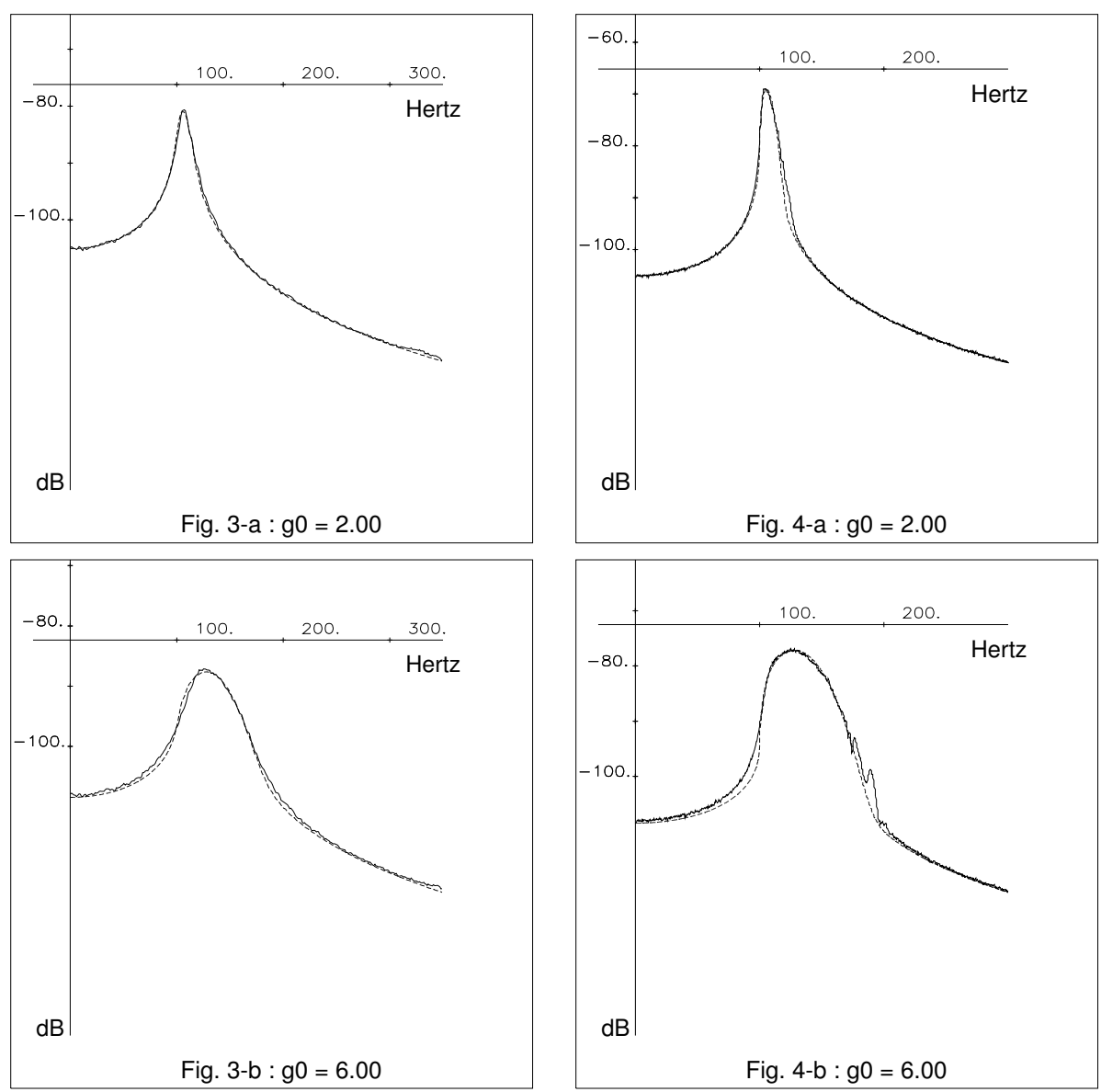

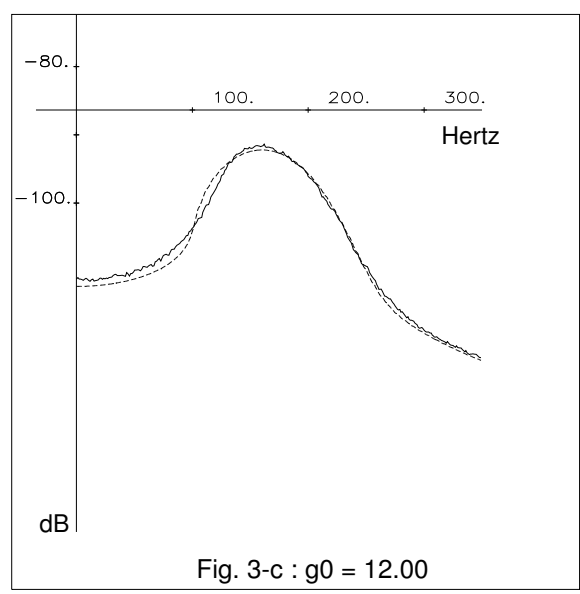

Fig. 3 : CASE 1

numerical simulation

SLM with random parameters

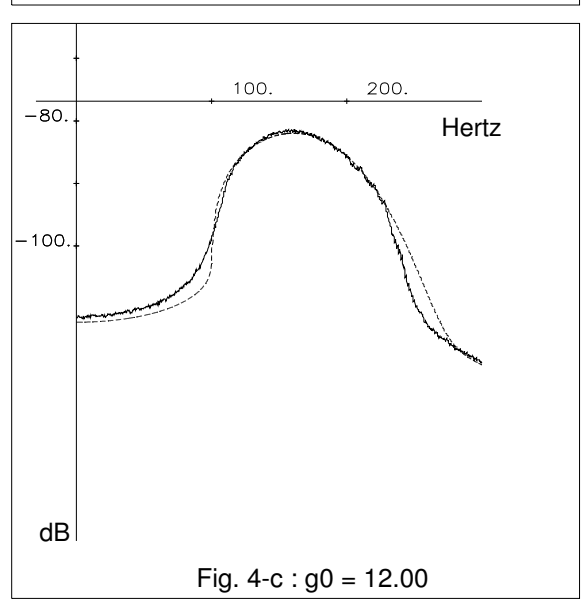

Fig. 4 : CASE 2

numerical simulation

SLM with random parameters

Proba. Eng. Mech. 

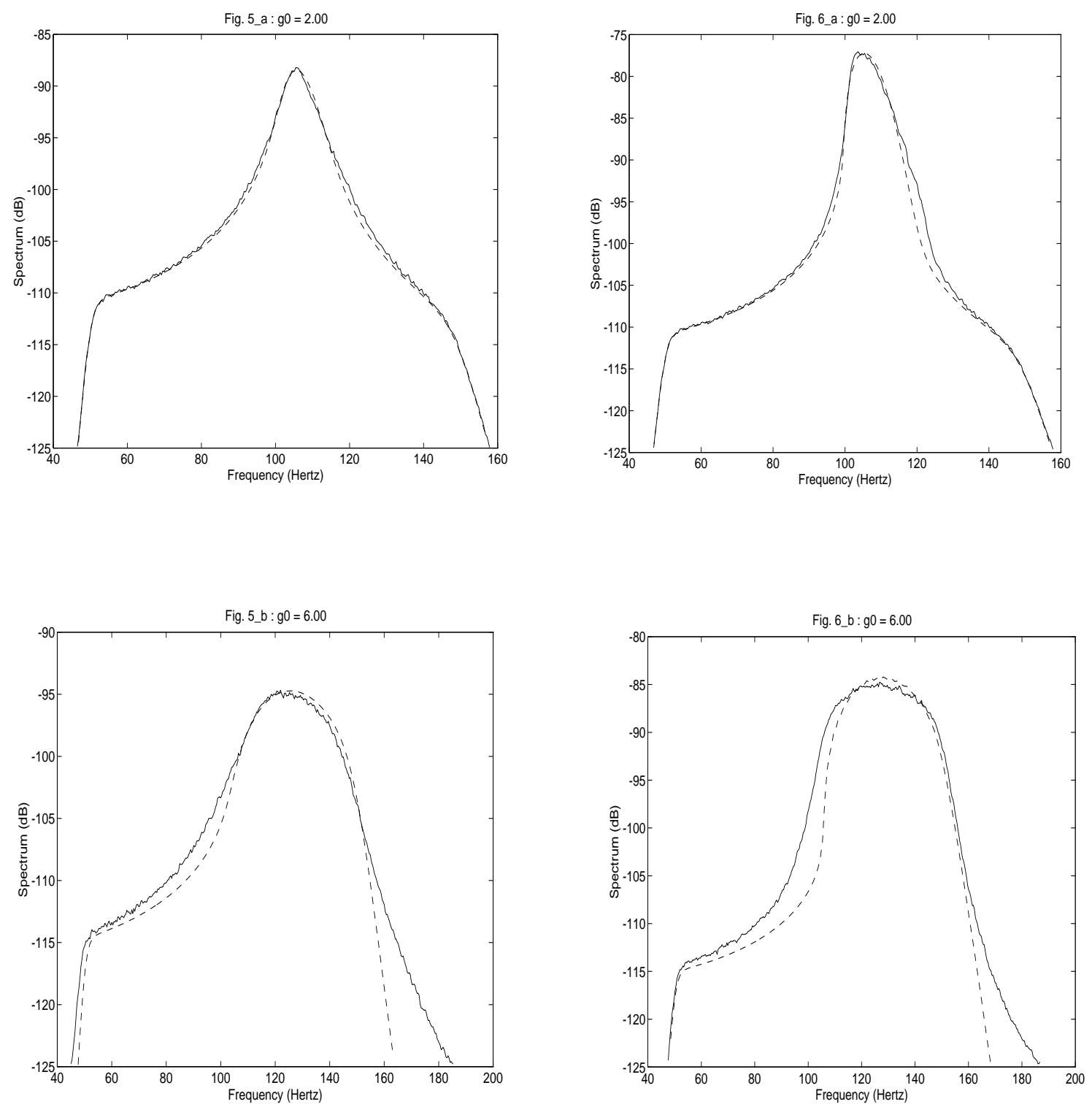

Fig. 5 : CASE 1

experimental spectrum

Fig. 6 : CASE 2 identified spectrum

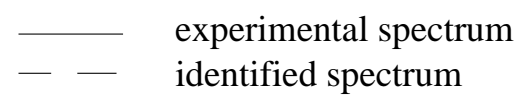

Proba. Eng. Mech. 